\title{
Arithmetical equivalents for a remarkable identity between theta functions.
}

By

E. 'T. Bell.

1. Except when $a=\not b=y=1$, or in the cases derived immediately from this by the transformation of the first order, Jacobi's fundamental identity for the multiplication of four thets functions ${ }^{1}$ ) at once expresses $\vartheta_{1}^{\prime} \vartheta_{x}(x+y) \vartheta_{p}(y+z) \vartheta_{y}(z+x)$ in terms of sums of products of theta functions. In the excepted cases the corresponding expressions are found from a remarkable identity first obtained by Hermite ${ }^{\mathrm{y}}$ ):

$$
\begin{gathered}
-\vartheta_{0}^{\prime}(x+y+z) \vartheta_{0}(x) \vartheta_{0}(y) \vartheta_{0}(z)+\vartheta_{0}(x+y+z) \vartheta_{0}^{\prime}(x) \vartheta_{0}(y) \vartheta_{0}(z) \\
(1)+\vartheta_{0}(x+y+z) \vartheta_{0}(x) \vartheta_{0}^{\prime}(y) \vartheta_{0}(z)+\vartheta_{0}(x+y+z) \vartheta_{0}(x) \vartheta_{0}(y) \vartheta_{0}^{\prime}(z) \\
=\vartheta_{1}^{\prime} \vartheta_{1}(x+y) \vartheta_{1}(y+z) \vartheta_{1}(z+x)
\end{gathered}
$$

which, as will be noticed, contains also the first derivatives of the thetas with respect to their arguments. This identity, although not included in Jacobi's, nevertheless can be obtained very simply from it, as shown by Enneperi").

Either Jacobi s identity or (1) gives rise to a host of arithmetical equivalents; and it is clear that the consequences of the two identities will be distinct, at least under elementary transformations. By an arithmetical equivalent of (1) we mean a relation between integers which implies (1) and which is implied by (1). We proceed presently to the derivation of three such equivalents, and it will be evident from the manner in which they are obtained that these are but three of a great many that may be found in essentially the same way. From the above definition of an equivalent it is obvious that ultimately all equivalents

1) Werke, 1, pp. 506, 509.

3) (Xomptes Rendus. Paria, 85 (1877), p. 731 .

) Mathematische Annalen, 17 (1380), p. 213. 
of (1) must express the same fact concerning integers. In particular it must be possible to transform any given equivalent into any other by purely arithmetical processes. These transformations in the majority of cases present considerable difficulty, and we make no attempt to exhibit them. It is in fact a point of interest in this kind of work that apparently unrelated arithmetical truths should appear from the standpoint of elementary analysis merely as different aspects of the same thing, in this case of the identity (1). The arithmetical results have been verified. numerically.

2. Changing $q$ into $-q$ in $(1)$ we get

$-\vartheta_{3}^{\prime}(x+y+z) \vartheta_{3}(x) \vartheta_{3}(y) \vartheta_{3}(z)+\vartheta_{3}(x+y+z) \vartheta_{3}^{\prime}(x) \vartheta_{3}(y) \vartheta_{3}(z)$ $(2)+\vartheta_{3}(x+y+z) \vartheta_{3}(x) \vartheta_{3}^{\prime}(y) \vartheta_{3}(z)+\vartheta_{3}(x+y+z) \vartheta_{3}(x) \vartheta_{3}(y) \vartheta_{3}^{\prime}(z)$ $=\vartheta_{1}^{\prime} \vartheta_{1}(x+y) \vartheta_{1}(y+z) \vartheta_{1}(z+x)$

and hence, on equating coefficients of like powers of $q$,

$$
\begin{aligned}
& \sum\left(l_{1}-l_{2}-l_{3}-l_{4}\right) \sin \left\{\left(l_{1}+l_{2}\right) x+\left(l_{1}+l_{3}\right) y+\left(l_{1}+l_{4}\right) z\right\} \\
= & \sum(-1)^{\frac{1}{2}\left(m_{1} m_{2} m_{3} m_{2}-1\right)} m_{1} \sin \left\{\left(m_{2}+m_{4}\right) x+\left(m_{2}+m_{3}\right) y+\left(m_{3}+m_{4}\right) z\right\}
\end{aligned}
$$

where the summations extend to all even integers $l_{i} \gtreqless 0$ and to all odd integers $m_{i} \gtrless 0(i=1,2,3,4)$ that satisfy

$$
4 m=l_{1}^{2}+l_{2}^{2}+l_{3}^{2}+l_{4}^{2}=m_{1}^{2}+m_{2}^{2}+m_{3}^{2}+m_{4}^{2},
$$

in which $m$ is odd and constant.

Let now $g(u, v, w)$ denote a function which takes a single definite value whenever $u, v, w$ are integers $\gtreqless 0$, and which is subject only to the conditions

$$
g(u, v, w)=-g(-u,-v,-w), g(0,0,0)=0 .
$$

We emphasize that beyond these restrictions, $g$ is general in the widest sense. Then, as a special consequence of a theorem on trigonometric identities established elsewhere ${ }^{4}$ ), we can infer from the identity in $x, y, z$,

$$
\sum_{i} p_{i} \sin \left(a_{i} x+b_{i} y+c_{i} z\right)=0
$$

in which $p_{i}, a_{i}, b_{i}, c_{i}$ are integers $\gtreqless 0$, the following,

$$
\sum_{i} p_{i} g\left(a_{i}, b_{i}, c_{i}\right)=0 \text {. }
$$

Hence, the summations being as in (3), we may assert that (3) implies

$$
\begin{aligned}
& \sum\left(l_{1}-l_{2}-l_{3}-l_{4}\right) g\left(l_{1}+l_{2}, l_{1}+l_{3}, l_{1}+l_{4}\right) \\
= & \sum(-1)^{\frac{1}{2}\left(m_{1} m_{2} m_{3} m_{1}-1\right)} m_{1} g\left(m_{2}+m_{4}, m_{2}+m_{3}, m_{3}+m_{4}\right),
\end{aligned}
$$

4) Transactions of the Amorican Math. Socioty, 2: (1921). p. j. 
Conversely, it is obvious that (6) implies (3), which evidently implies (2) and (1). For if with Jacobi (loc. cit. p. 501) we take as definitions of the theta functions their expansions as power series in $q$, it is clear that, as a relation between series, (3) implies (2). Hence (2), (6) are equivalent.

3. The very light conditions (5) can be satisfied in many ways which still leave a wide margin of generality, and which severally lead to results of arithmetical interest. Let $(x, y, z)$ denote any permutation of $(u, v, w)$. Then $g(u, v, w)$ in (6) may be replaced by any one of the following, in which the appropriate restrictions are indicated by the equations:

$g_{1}(x, y, z)=-g_{1}(-x, y, z)=g(x,-y,-z), g(0, y, z)=0$;

$g_{2}(x, y, z)=g_{2}(-x, y, z)=-g_{2}(x,-y,-z), g_{2}(x, 0,0)=0$;

$g_{8}(x, y, z)=-g_{3}(-x, y, z)=g_{3}(x,-y, z)=g_{3}(x, y,-z) g(0, y, z)=0$;

$g_{4}(x, y, z)=-g_{1}(-x, y, z)=-g_{4}(x,-y, z)=-g_{4}(x, y,-z)$,

$g_{4}(0, y, z)=g_{4}(x, 0, z)=g_{4}(x, y, 0)=0$;

$g_{5}(x, y)=-g_{5}(-x, y)=g_{5}(x,-y), g_{5}(0, y)=0$;

$g_{6}(x)=-g_{6}(-x), g_{6}(0)=0$.

From these we see by inspection the forms of the most general polynomials satisfying the conditions rimposed upon $g(u, v, w)$. Let the $a_{i}, b_{i}, c_{i}$ denote integers $>0$, the $p_{i}, q_{i}, r_{i}, s_{i}$ constants other than zero. Then the polynomials are

$$
\begin{aligned}
& \sum_{i} p_{i}\left(q_{i} x\right)^{2 a_{i}-1}\left(r_{i} y+s_{i} z\right)^{2 b_{i}}, \\
& \sum_{i} p_{i}\left(q_{i} x\right)^{2 a_{i}}\left(r_{i} y+s_{i} z\right)^{2 b_{i}-1}, \\
& \sum_{i} p_{i}\left(q_{i} x\right)^{2 a_{i}-1}\left(r_{i} y\right)^{2 b_{i}}\left(s_{i} z\right)^{2 c_{i}} \\
& \sum_{i} p_{i}\left(q_{i} x\right)^{2 a_{i}-1}\left(r_{i} y\right)^{2 b_{i}-1}\left(s_{i} z\right)^{2 a_{i}-1}, \\
& \sum_{i} p_{i}\left(q_{i} x\right)^{2 a_{i}-1}\left(r_{i} y\right)^{2 b_{i}}, \\
& \sum_{i} p_{i}\left(q_{i} x\right)^{2 a_{i}-1} .
\end{aligned}
$$

The functions $g_{1}, \ldots, g_{6}$ are general beyond the restrictions stated.

4. Let us examine the kind of information furnished by (6) in special cases. For this it will be sufficient to consider one simple example, $g(u, v, w)=u v w$. Taking this from of $g(u, v, w)$ in (6), and omitting the terms which destroy one another owing to differences of sign when the summations are performed over all solutions of (4), we find

$$
\sum\left[l_{1}^{4}-l_{1}^{2}\left(l_{2}^{3}+l_{3}^{2}+l_{1}^{2}\right)\right]=2 \sum(-1)^{\frac{1}{2}\left(m_{2} m_{2} m_{3} m_{4}-1\right)} m_{1} m_{2} m_{8} m_{4} .
$$


To reduce this, let $N_{r}(n, s)$ denote the total number of representations of $n$ as a sum of $r$ squares precisely $s$ of which are odd, and write $\zeta_{r}(n)$ for the sum of the $r$ th powers of all the divisors of $n$. Then by well known theorems we have

$$
N_{4}(4 m, 0)=8 \zeta_{1}(m)=1 / 7 \zeta_{1}(4 m)
$$

since $m$ is odd; and hence from (7), on using $4 m-l_{1}^{2}=l_{2}^{2}+l_{8}^{2}+l_{4}^{3}$ from (4), we find

$$
7 \sum\left[l_{1}^{4}-(-1)^{\frac{1}{2}\left(m_{1} m_{2} m_{3} m_{4}-1\right)} m_{1} m_{9} m_{3} m_{4}\right]=n^{2} \zeta_{1}(n),
$$

in which $n \equiv 4 \bmod 8$, and the summation extends to all aven $l_{1} \gtreqless 0$ and to all odd $m_{i} \gtrless 0$ satisfying

$$
n=l_{1}^{3}+l_{2}^{2}+l_{3}^{2}+l_{4}^{3}=m_{1}^{2}+m_{3}^{2}+m_{3}^{2}+m_{4}^{2},
$$

$l_{3}, l_{3}, l_{4} \gtreqless 0$ being even. In (8) we may replace $l_{1}$ by $l_{9}^{\prime}, l_{3}$ or $l_{4}$ from symmetry, and hence by addition of the results, $(8)$ is the same as

$$
\text { (9) } 7 \sum\left[l_{1}^{4}+l_{2}^{4}+l_{3}^{4}+l_{1}^{4}-4(-1)^{\frac{1}{2}\left(m_{1} m_{2} m_{3} m_{4}-1\right)} m_{1} m_{3} m_{3} m_{4}\right]=4 n^{2} \zeta_{1}(n) \text {. }
$$

From this and

$$
n^{2}-l_{1}^{4}-l_{2}^{4}-l_{3}^{4}-l_{4}^{4}=2\left(l_{1}^{2} l_{2}^{2}+l_{1}^{a} l_{3}^{2}+\ldots+l_{3}^{3} l_{4}^{2}\right)
$$

we find a similar relation involving $l_{1}^{2} l_{2}^{2}+\ldots+l_{3}^{2} l_{4}^{3}$; and hence by symmetry since $\sum l_{1}^{2} l_{2}^{2}=\ldots=\Sigma l_{3}^{3} l_{4}^{2}$, we get from (8) the corresponding relation in which $l_{1}^{4}$ is replaced by $l_{1}^{2} l_{2}^{2}$. It is interesting to compare (8) with a somewhat similar theorem stated without proof by Liouville ${ }^{5}$ ), and proved arithmetically by Stern ${ }^{8}$ ).

5. The identity (2) and its equivalent (6) are related through the representations of $4 \mathrm{~m}$ in the pair of arithmetical forms given in (4). The further, less obvious, equivalents of (1) or (2) are related through one or more quadratic forms, corresponding to (4), which no longer are exclusively sums of squares as in the first case. A great variety of these more interesting equivalents can be obtained by using, as below, the arithmetical developments for the doubly periodic functions of the second kind, $\varphi_{\alpha \beta \gamma}(u, v)$, defined by

$$
\varphi_{\alpha \beta \gamma}(u, v)=\frac{\vartheta_{1}^{\prime} \vartheta_{\alpha}(u, v)}{\vartheta_{\beta}(u) \vartheta_{y}(v)} .
$$

These functions, together with (in some cases) the doubly periodic functions of the third kind, are imported into (1) by multiplying it throughout by the same theta quotient and then rearranging the result. The process

5) Journal des Mathématiques, (2) 3 (1858), pp. 357-360.

6) Journal für d. r. u. a. Math., 105 (1889), pp. 251-262. 
will be sufficiently clear from the two equivalents which we derive next. We shall require the following development,

$$
\varphi_{\text {1mo }}(u, v)=4 \sum q^{\frac{1}{2} m}\left[\sum \sin (d x+\delta y)\right],
$$

in which the outer summation refers to all odd $m=1,3,5, \ldots$, and the inner to all divisors $d, \delta>0$ of $m$ such that $d \delta=m$. The sum enclosed in [] is the coefficient of $q^{\frac{1}{2} m}$, and similarly in writing other series. This series is due to Hermite ${ }^{7}$ ) (in a slightly different form).

6. Dividing (2) throughout by $\vartheta_{3}(x) \vartheta_{3}(y)$ we get

11) $\left[\vartheta_{3}(x+y+z) \vartheta_{3}^{\prime}(z)-\vartheta_{3}^{\prime}(x+y+z) \vartheta_{3}(z)\right]+\vartheta_{3}\left(x+y+z \vartheta_{3}(z)\left[\frac{\theta_{3}^{\prime}(x)}{\vartheta_{3}(x)}+\frac{\theta_{3}^{\prime}(y)}{\vartheta_{3}(y)}\right]\right.$ $=\vartheta_{1}(x+z) \vartheta_{1}(y+z) \varphi_{133}(x, y)$

The development of $\varphi_{133}(x, y)$ is found from that of $q_{100}(x, y)$ by changing $x, y$ into $x+\frac{\pi}{2}, y+\frac{\pi}{2}$,

$$
\begin{aligned}
\varphi_{133}(x, y) & =-\psi_{100}\left(x+\frac{\pi}{2}, y+\frac{\pi}{2}\right) \\
& =4 \sum q^{\frac{1}{2} m}\left[(-1)^{\frac{1}{2}(m-1)} \sum \sin (d x+\lambda y)\right] ;
\end{aligned}
$$

and from the classical series for the logarithmic derivative of $\vartheta_{0}(u)$ we find at once

(13) $\frac{\theta_{0}(u)}{\vartheta_{0}(u)}=4 \sum q^{n}\left[\sum \sin 2 t x\right], \quad \frac{\theta_{3}^{\prime}(u)}{\vartheta_{3}(u)}=4 \sum q^{n}\left[(-1)^{n} \sum \sin 2 t x\right]$,

where the outer summation extends to all positive integers $n=1,2,3,4, \ldots$, and the inner to all divisors $t$-of $n$ such that $\tau$ is odd, $t$ odd or even according as $n$ is odd or even, and $n=t \tau$. On substituting for the functions $\vartheta_{3}(u), \vartheta_{3}^{\prime}(u)$ their developments in (11) and likewise for the remaining functions from (12), (13), we find that the general coefficients of the general power of $q$ in (11) are summed with respect to all solutions of the following system in which $n$ is an arbitrary integer $>0$ :

$$
4 n=l_{1}^{2}+l_{2}^{2}=l_{1}^{\prime 2}+l_{2}^{\prime 2}+4 t_{3}^{\prime} \tau_{3}^{\prime}=m_{1}^{\prime \prime 2}+m_{2}^{\prime \prime 2}+2 d_{3}^{\prime \prime} \delta_{3}^{\prime \prime},
$$

in which $l_{1}, l_{3}, l_{1}^{\prime}, l_{2}^{\prime} \gtreqless 0$ are even, $m_{1}^{\prime \prime}, m_{2}^{\prime \prime} \gtrless 0$ are odd, $\tau_{3}^{\prime}, d_{3}^{\prime \prime}, \delta_{3}^{\prime \prime}>0$ are odd, and $t_{3}^{\prime}>0$ is odd or even. For simplicity in writing (15) put $n_{3}^{\prime}=t_{3}^{\prime} t_{3}^{\prime}, m_{3}^{\prime \prime}=d_{3}^{\prime \prime} \delta_{3}^{\prime \prime}$. Then, proceeding as in the derivation of $(6)$, we infer that (11) is equivalent to

?) Oeuwres, 4. p. 199. 


$$
\begin{aligned}
& \sum l_{2}\left[g\left(l_{2}, l_{3}, l_{1}+l_{2}\right)-g\left(l_{1}, l_{1}, l_{1}+l_{2}\right)\right] \\
& \text { (15) } \\
& +4 \sum(-1)^{n_{3}^{\prime}}\left[g\left(l_{1}^{\prime}+2 t_{3}^{\prime}, l_{1}^{\prime}, l_{1}^{\prime}+l_{2}^{\prime}\right)-g\left(l_{1}^{\prime}, l_{1}^{\prime}+2 t_{3}^{\prime}, l_{1}^{\prime}+l_{2}^{\prime}\right)\right]
\end{aligned}
$$

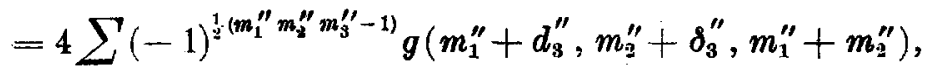

the summations extending to all solutions of the prescribed kinds for $n$ fixed of the system (14). As before it follows that (2), (15) are equivalent.

7. The next equivalent is of particular interest because it involves also the divisors $d, \delta$ of the fixed odd integer $m$ which is separated into quadratic forms as follows:

$$
m=d \delta=d_{1} \delta_{1}+d_{3} \delta_{2}+d_{3} \delta_{3}=2 t_{1}^{\prime} \tau_{1}^{\prime}+d_{2}^{\prime} \delta_{2}^{\prime}
$$

in which $d, \delta, d_{1}, \delta_{1}, d_{3}, \delta_{2}, d_{x}, \delta_{3}, \tau_{1}^{\prime}, d_{2}^{\prime}, \delta_{2}^{\prime}>0$ are odd, $t_{1}^{\prime}>0$ is odd or even.

Divide (1) throughout by

$$
\vartheta_{0}(x) \vartheta_{0}(y) \vartheta_{0}(z) \vartheta_{0}(x+y+z)
$$

and write the result in the form

$$
\begin{aligned}
& \varphi_{100}(x+y+z,-z) \varphi_{100}(y, z) \varphi_{100}(x, z) \\
& +\frac{\vartheta_{1}^{\prime 2}}{\vartheta_{0}^{2}(z)}\left[\frac{\vartheta_{0}^{\prime}(x+y+z)}{\vartheta_{0}(x+y+z)}-\frac{\vartheta_{0}^{\prime}(x)}{\vartheta_{0}^{\prime}(x)} \frac{\vartheta_{0}^{\prime}(y)}{\vartheta_{0}(y)}\right]=\frac{\vartheta_{1}^{\prime 2} y_{0}^{\prime}(z)}{\vartheta_{0}^{3}(z)} .
\end{aligned}
$$

To give a simple equivalent of this we require in addition to the series $(10),(13)$ the following

$$
\begin{gathered}
\frac{\theta_{1}^{\prime 2}}{\vartheta_{0}^{2}(u)}=4 \sum q^{\frac{1}{2} m}\left[\sum d \cos (d-\delta) u\right], \\
\frac{\vartheta_{1}^{\prime 2} \vartheta_{0}^{\prime}(u)}{\vartheta_{0}^{3}(u)}=2 \sum q^{\frac{1}{2} m}\left[\sum\left(d^{3}-m\right) \sin (d-\delta) u\right],
\end{gathered}
$$

in which the outer summations are as defined for (10). We need not stop here to give the derivations of $(18),(19)$, which can be checked in various ways, but remark that both can be obtained quite simply from (10). From (17) by means of (10), (13), (18), (19) we find the following equivalent of $(1)$ in the same way as before: 
152 E. T. Bell. A rithm. equid. f. a remark. ident. between theta functions.

(20)

$$
\begin{aligned}
8 \sum & {\left[g\left(d_{1}-d_{3}, d_{1}+d_{2}, d_{1}-\delta_{1}+\delta_{2}-\delta_{2}\right)\right.} \\
& +g\left(d_{1}+d_{3}, d_{2}-d_{2}, d_{1}-\delta_{1}-\delta_{2}+\delta_{3}\right) \\
& -g\left(d_{1}+d_{3}, d_{1}+d_{2}, d_{1}-\delta_{1}+\delta_{2}-\delta_{3}\right) \\
& \left.-g\left(d_{1}-d_{3}, d_{1}-d_{2}, d_{1}-\delta_{1}-\delta_{2}-\delta_{3}\right)\right] \\
4 \sum d_{2}^{\prime}[ & g\left(2 t_{1}^{\prime}, 2 t_{1}^{\prime}, 2 t_{1}^{\prime}+d_{2}^{\prime}-\delta_{2}^{\prime}\right)+g\left(2 t_{1,3}^{\prime} 2 t_{1}^{\prime}, 2 t_{1}^{\prime}-d_{2}^{\prime}+\delta_{2}\right) \\
- & g\left(2 t_{1}^{\prime}, 0, d_{2}^{\prime}-\delta_{2}^{\prime}\right)-g\left(2 t_{1}^{\prime}, 0,-d_{2}^{\prime}+\delta_{2}^{\prime}\right) \\
& \left.-g\left(0,2 t_{1}^{\prime}, d_{2}^{\prime}-\delta_{2}^{\prime}\right)-g\left(0,2 t_{1}^{\prime},-d_{2}^{\prime}+\delta_{2}^{\prime}\right)\right] \\
= & \sum\left(d^{2}-m\right) g(0,0, d-\delta),
\end{aligned}
$$

the summation extending for $m$ constant to all solutions of (16) of the kinds prescribed.

(Eingegangen ain 11. August 1921.) 